\title{
Beam focusing limitation from synchrotron radiation in two dimensions
}

\author{
O. R. Blanco, ${ }^{1,2}$ R. Tomás,,$^{2, *}$ and P. Bambade ${ }^{1}$ \\ ${ }^{1}$ LAL, Université Paris-Sud, CNRS/IN2P3, Orsay 91898, France \\ ${ }^{2}$ CERN, Geneva 1211, Switzerland \\ (Received 18 September 2015; published 19 February 2016)
}

\begin{abstract}
The Oide effect considers the synchrotron radiation in the final focusing quadrupole, and it sets a lower limit on the vertical beam size at the interaction point, particularly relevant for high-energy linear colliders. The theory of the Oide effect was derived considering only the second moment of the radiation in the focusing plane of the magnet. This article addresses the theoretical calculation of the radiation effect on the beam size considering the first and second moments of the radiation and both focusing and defocusing planes of the quadrupole. The effect for a Gaussian beam is referred to as 2D-Oide; however, an alternative beam size figure is given that could represent better the effect on the minimum achievable $\beta_{y}^{*}$. The CLIC $3 \mathrm{TeV}$ final quadrupole (QD0) and beam parameters are used to compare the theoretical results from the Oide effect and the 2D-Oide effect with particle tracking in PLACET. The 2D-Oide effect is demonstrated to be important, as it increases by $17 \%$ the contribution to the beam size. Further insight into the aberrations induced by the synchrotron radiation opens the possibility to partially correct the 2D-Oide effect with octupole magnets. A beam size reduction of $4 \%$ is achieved in the simplest configuration, using a single octupole.
\end{abstract}

DOI: 10.1103/PhysRevAccelBeams.19.021002

\section{INTRODUCTION}

Synchrotron radiation in a focusing quadrupole magnet of length $L$ and gradient $k$, schematically represented in Fig. 1, changes the energy of the particle and modifies the focusing effect. This results in a limit on the minimum beam size at the interaction point (IP) located at a distance $l^{*}$ from the quadrupole. This is referred to as the Oide effect [1].

The beam size growth due to radiation is added quadratically to the linear beam size $\sigma_{0}^{2}=\epsilon \beta^{*}$, where $\beta^{*}$ represents the optical beta function at the IP and $\epsilon$ is the emittance. Therefore, $\sigma^{2}=\sigma_{0}^{2}+\sigma_{\text {Oide }}^{2}$, where the beam size contribution from the Oide effect is [1]

$$
\sigma_{\text {Oide }}^{2}=\frac{110}{3 \sqrt{6 \pi}} r_{e} \frac{\lambda_{e}}{2 \pi} \gamma^{5} F\left(\sqrt{k} L, \sqrt{k} l^{*}\right)\left(\frac{\epsilon}{\beta^{*}}\right)^{5 / 2}
$$

with

$$
\begin{aligned}
F\left(\sqrt{k} L, \sqrt{k} l^{*}\right)= & \int_{0}^{\sqrt{k} L}\left|\sin \phi+\sqrt{k} l^{*} \cos \phi\right|^{3} \\
& \times\left[\int_{0}^{\phi}\left(\sin \phi^{\prime}+\sqrt{k} l^{*} \cos \phi^{\prime}\right)^{2} d \phi^{\prime}\right]^{2} d \phi,
\end{aligned}
$$

\footnotetext{
*rogelio.tomas@cern.ch
}

Published by the American Physical Society under the terms of the Creative Commons Attribution 3.0 License. Further distribution of this work must maintain attribution to the author(s) and the published article's title, journal citation, and DOI. and $\lambda_{e}$ is the Compton wavelength of the electron, $r_{e}$ is the classical electron radius, and $\gamma$ is the relativistic factor.

The primitive of the double integral used to calculate $F$ in Eq. (2) is derived analytically in [2] with the goal to increase the computational calculation speed. It has been included in MAPCLASS2 [3-6] to be used in lattice design and optimization.

Although the total contribution to beam size depends on the lattice and beam parameters, the minimum achievable beam size is given by [1]

$$
\sigma_{\min }=\left(\frac{7}{5}\right)^{1 / 2}\left[\frac{275}{3 \sqrt{6 \pi}} r_{e} \frac{\lambda_{e}}{2 \pi} F\left(\sqrt{K} L, \sqrt{K} l^{*}\right)\right]^{1 / 7}\left(\epsilon_{N}\right)^{5 / 7},
$$

where $\epsilon_{N}=\gamma \epsilon$ is the normalized emittance, showing the independence from beam energy.

Table I shows the relevant parameters of the last vertically focusing magnet QD0 $(k, L)$, the beam and lattice optics $\left(\epsilon_{N}, \gamma, \sigma_{0}, l^{*}\right)$ for the two main linear collider projects ILC $500 \mathrm{GeV}$ [7] and CLIC at $500 \mathrm{GeV}$ and $3 \mathrm{TeV}$ [8]. These are used to show that the contribution of the Oide effect to the vertical beam size is significant only

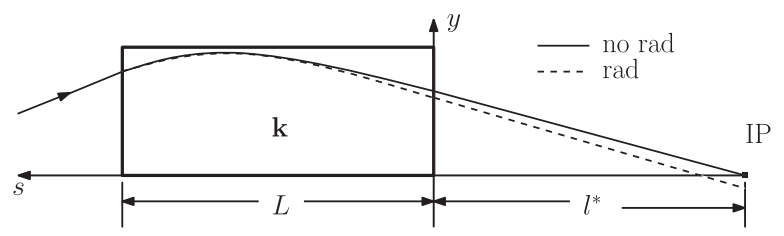

FIG. 1. The solid and dashed lines represent the particle trajectory without and with radiation, respectively. 
TABLE I. Vertical beam size and radiation beam size contribution for three lattices. $\epsilon_{N}$ is the normalized emittance, $\epsilon_{N}=\gamma \epsilon$.

\begin{tabular}{lcccccccccc}
\hline \hline Lattice & $\epsilon_{N}[\mathrm{~nm}]$ & $\gamma\left[10^{3}\right]$ & $\sigma_{0}[\mathrm{~nm}]$ & $k\left[\mathrm{~m}^{-2}\right]$ & $L[\mathrm{~m}]$ & $l^{*}[\mathrm{~m}]$ & $F$ & $\sigma_{\text {Oide }}[\mathrm{nm}]$ & $\sigma[\mathrm{nm}]$ & $\sigma_{\text {min }}[\mathrm{nm}]$ \\
\hline CLIC 3 TeV & 20 & 2935.0 & 0.70 & 0.116 & 2.73 & 3.5 & 4.086 & 0.87 & 1.10 & 1.00 \\
CLIC 500 GeV & 25 & 489.2 & 2.3 & 0.077 & 3.35 & 4.3 & 4.115 & 0.08 & 2.3 & 1.17 \\
ILC 500 GeV & 40 & 489.2 & 5.7 & 0.170 & 2.20 & 4.3 & 9.567 & 0.04 & 5.7 & 1.85 \\
\hline \hline
\end{tabular}

for CLIC $3 \mathrm{TeV}$. Also, columns $\sigma$ and $\sigma_{\min }$ show that the final vertical beam size is comparable to the minimum achievable.

If none of the beam parameters or $l^{*}$ is to be changed, then $F$ can be used as a figure of merit of the quadrupole setup as it is calculated only from $k, L$, and $l^{*}$, where the target is to reduce $F$ as much as possible. The standard procedure of reducing $F$ is by increasing the length of the quadrupole and reducing its gradient. In the following, this is illustrated for CLIC $3 \mathrm{TeV}$.

The relative increase in the beam size due to the Oide effect is given by

$$
\sigma / \sigma_{0}=\sqrt{1+\sigma_{\text {Oide }}^{2} / \sigma_{0}^{2}}
$$

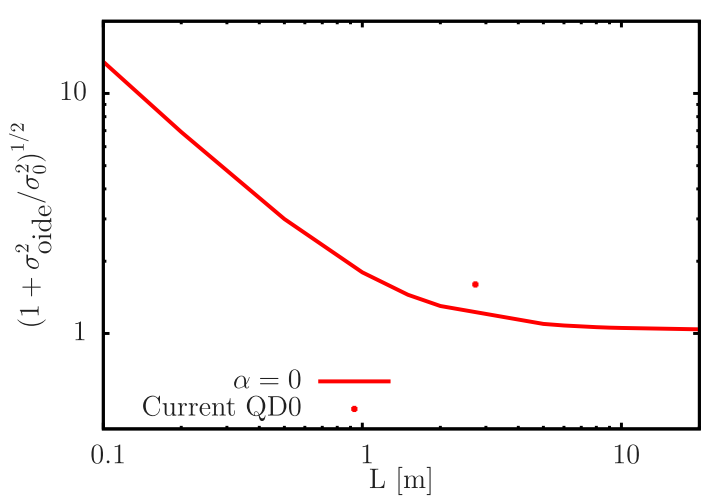

(a)

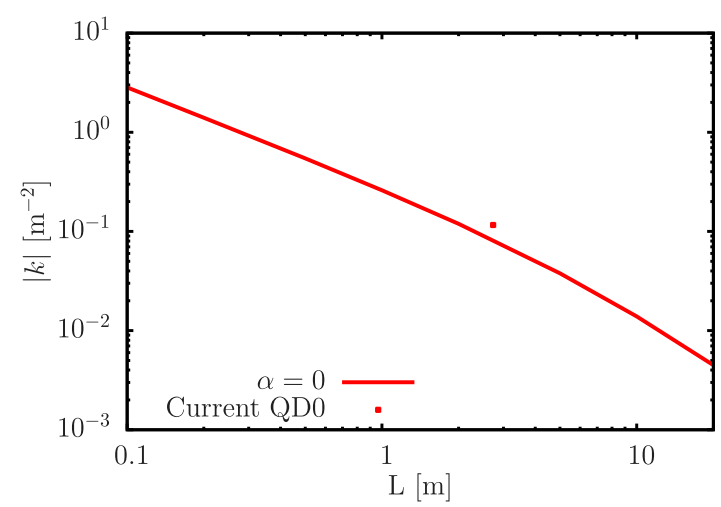

(b)

FIG. 2. Oide effect beam size contribution for CLIC $3 \mathrm{TeV}$ design parameters. (a) Total beam size normalized to the designed linear beam size as a function of the quad length for the minimum focusing $k$ and the current QD0. (b) $k$ in the two previous cases.
Figure 2(a) shows the relative increase due to the Oide effect when $k$ is set to cancel the optics function alpha, $\alpha$, just at the quadrupole opposite face to the IP, the particle input. This is done to set an absolute minimum in the Oide effect out of reach for realistic final doublet (FD) designs. Figure 2(b) shows the $k$ values previously mentioned.

The current QD0 almost doubles the vertical beam size. It might be possible to reduce $\sigma_{\text {Oide }}^{2}$ by a small factor by increasing the current quadrupole length and possibly using a lower $k$; however, this solution also points to increasing the lattice length. This could also generate difficulties in lattice design because of chromaticity and magnet stabilization. Quad lengths larger than $10 \mathrm{~m}$ do not lead to further significant improvements.

\section{2D-OIDE EFFECT}

In order to gain an understanding for the relevant case of CLIC at $3 \mathrm{TeV}$, Sec. II is dedicated to the theoretical derivation of the effect of radiation including the horizontal beam size and optics lattice parameters (2D-Oide) and comparing results with particle tracking. This leads to a possible way to mitigate the effect, alternative to enlarging QD0, consisting in removing the correlation at the IP via normal octupole magnets. This is tested for the simplest case of one octupole magnet as an example.

\section{A. Analytical derivation}

$E_{0}$ being the nominal energy of the beam and $u$ the energy of the photon radiated, in a first-order approximation where $u \ll E_{0}$, the total effect of radiation in the vertical displacement at the IP is calculated integrating along the magnet length as

$$
\Delta y=\int_{0}^{L} \int_{0}^{s} f_{y}^{2}\left(\sqrt{k} s_{1}\right) \frac{u}{E_{0}} y_{0}^{\prime *} d s_{1} d s,
$$

where the inner integral represents the kick given by the radiated photon at a certain location $s$ propagated to the IP assuming a strong focusing in the vertical plane $\left(l^{*} \gg \beta_{y}^{*}\right)[1]$. $y_{0}^{\prime *}$ is the vertical particle angle at the IP without radiation, and

$$
f_{y}(\phi)=\sin \phi+\sqrt{k} l^{*} \cos \phi .
$$

Because $u$ is much smaller than $E_{0}$ in Eq. (5), it is still possible to use the result in [9] for the average energy loss per unit length in the magnet $\bar{u}$ and its second moment $\overline{u^{2}}$ shown in Eqs. (7) and (8): 


$$
\begin{aligned}
& \frac{\bar{u}}{E_{0}}=\frac{2}{3} r_{e} \frac{\gamma^{3}}{\rho^{2}}, \\
& \frac{\overline{u^{2}}}{E_{0}}=\frac{55}{24 \sqrt{3}} r_{e} \frac{\lambda_{e}}{2 \pi} \gamma^{5} \frac{1}{|\rho|^{3}} .
\end{aligned}
$$

This allows one to calculate two important values: the mean effect of the radiation in the trajectory of one particle along the magnet $\overline{\Delta y}$ and its second moment $\overline{(\Delta y)^{2}}$.

The calculation derived by Oide considers the beam size in the defocusing plane of the final quadrupole to be negligible; however, if the beam size in the defocusing plane is considered, the bending radius of curvature $\rho$ in the lens is given by

$$
\frac{1}{|\rho(s)|}=|k| \sqrt{x^{2}(s)+y^{2}(s)}
$$

and Fig. 3 shows schematically the particle trajectory. As shown in Eqs. (7) and (8), the radius of curvature depending on both transversal planes has a direct impact on the radiation.

In addition, assuming $l^{*} \gg \beta_{x}^{*}$, the coordinates $x(s)$ and $y(s)$ in Eq. (9) can be calculated from the horizontal $\left(x_{0}^{*}\right)$ and vertical $\left(y_{0}^{\prime *}\right)$ angles at the IP as

$$
\begin{aligned}
& x(s)=-x_{0}^{\prime *} \frac{f_{x}(\sqrt{k} s)}{\sqrt{k}}, \\
& y(s)=-y_{0}^{\prime *} \frac{f_{y}(\sqrt{k} s)}{\sqrt{k}},
\end{aligned}
$$

where

$$
f_{x}(\phi)=\sinh \phi+\sqrt{k} l^{*} \cosh \phi .
$$

Combining the expressions in Eqs. (7), (9), (10), and (11), it is possible to average over the photon energy in Eq. (5), obtaining

$$
\overline{\Delta y}=a\left(y_{0}^{\prime *}\right)^{3}+b\left(x_{0}^{\prime *}\right)^{2} y_{0}^{\prime *},
$$

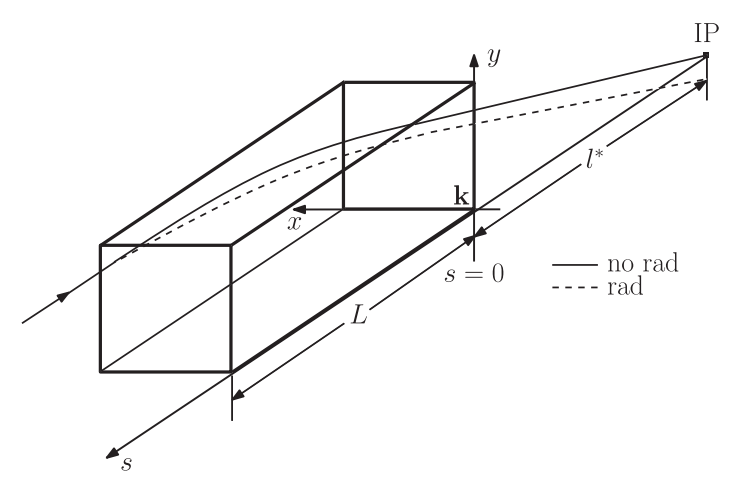

FIG. 3. The solid and dashed lines represent the particle trajectory without and with radiation, respectively. where

$$
\begin{aligned}
& a=\frac{2}{3} r_{e} \gamma^{3} \int_{0}^{\sqrt{k} L} f_{y}^{2}(\phi) F_{y}(\phi) d \phi=\frac{1}{3} r_{e} \gamma^{3} F_{y}^{2}(\sqrt{k} L), \\
& b=\frac{2}{3} r_{e} \gamma^{3} \int_{0}^{\sqrt{k} L} f_{x}^{2}(\phi) F_{y}(\phi) d \phi,
\end{aligned}
$$

and

$$
\begin{aligned}
F_{y}(\phi)= & \int_{0}^{\phi} f_{y}^{2}\left(\phi^{\prime}\right) d \phi^{\prime} \\
= & \frac{\phi}{2}\left[\left(\sqrt{k} l^{*}\right)^{2}+1\right]+\frac{\sin (2 \phi)}{4}\left[\left(\sqrt{k} l^{*}\right)^{2}-1\right] \\
& +\sqrt{k} l^{*} \sin ^{2} \phi .
\end{aligned}
$$

Equation (13) shows a cubic component and, in addition, a linear component in $y_{0}^{\prime *}$ whose magnitude depends on the horizontal angle at the IP, showing explicitly the correlation between the two planes when the beam size in the defocusing plane is not negligible.

As we are interested in the effect over an ensemble of particles, the expected value of a function $\Psi$ is defined as

$$
\langle\Psi\rangle=\int_{-\infty}^{\infty} \int_{-\infty}^{\infty} \Psi \Omega\left(x_{0}^{\prime *}, y_{0}^{\prime *}\right) d x_{0}^{\prime *} d y_{0}^{\prime *},
$$

where $\Omega$ represents a Gaussian distribution in both horizontal and vertical particle angles at the IP.

The average particle deviation due to radiation $\langle\overline{\Delta y}\rangle$ is equal to zero, but, in order to explore the correlation of the radiation with $y_{0}^{\prime *}$, the two components, $a$ and $b\left(x_{0}^{\prime *}\right)^{2}$, are evaluated further in this section using $\left\langle\left(x_{0}^{\prime *}\right)^{2}\right\rangle=\sigma_{x_{0}^{\prime *}}^{2}=\epsilon_{N x} /\left(\gamma \beta_{x}^{*}\right)$ and later compared with particle tracking results of CLIC $3 \mathrm{TeV}$ QD0.

$\left\langle(\overline{\Delta y})^{2}\right\rangle$ is given by

$$
\left\langle(\overline{\Delta y})^{2}\right\rangle=15 a^{2}\left(\frac{\epsilon_{y}}{\beta_{y}^{*}}\right)^{3}+3 b^{2}\left(\frac{\epsilon_{x}}{\beta_{x}^{*}}\right)^{2} \frac{\epsilon_{y}}{\beta_{y}^{*}}+6 a b\left(\frac{\epsilon_{y}}{\beta_{y}^{*}}\right)^{2} \frac{\epsilon_{x}}{\beta_{x}^{*}}
$$

and used in Sec. II C to calculate the maximum theoretical mitigation of the radiation effect and to evaluate the mitigation method.

In a similar way, the square of the vertical displacement in Eq. (5), $(\Delta y)^{2}$, is calculated combining Eqs. (8)-(11) by averaging over photons the second moment of the energy loss, resulting in Eq. (19):

$$
\begin{aligned}
\overline{(\Delta y)^{2}}= & \frac{55}{24 \sqrt{3}} r_{e} \frac{\lambda_{e}}{2 \pi} \gamma^{5}\left(y_{0}^{\prime *}\right)^{2} \\
& \times \int_{0}^{\sqrt{k} L}\left\{\left[y_{0}^{\prime *} f_{y}(\phi)\right]^{2}+\left[x_{0}^{\prime *} f_{x}(\phi)\right]^{2}\right\}^{3 / 2} F_{y}^{2}(\phi) d \phi .
\end{aligned}
$$


$\left\langle\overline{(\Delta y)^{2}}\right\rangle=\sigma_{\text {Oide }}^{2}$ when $\sigma_{x_{0}^{\prime *}}=0$. However, when $\sigma_{x_{0}^{\prime *}}$ is not zero, the expected value cannot be calculated analytically; it must be evaluated numerically, and it is referred to as $\sigma_{2 \mathrm{D} \text {-Oide }}^{2}$ below. This is also compared with particle tracking simulations in this section for the case of CLIC $3 \mathrm{TeV}$. An analytical approximation to the integrals over the particle distribution for $\sigma_{2 \mathrm{D} \text {-Oide }}$ has been derived in [10], Sec. 11.6.4, using power series.

To put aside the limitation in the analytic calculation of $\sigma_{2 \mathrm{D}-\text {-Oide, }}$, it is also possible to estimate the effect of radiation using the square of the expected absolute vertical displacement $\langle|\overline{\Delta y}|\rangle^{2}$. Having

$$
|\overline{\Delta y}|=a\left|y_{0}^{\prime *}\right|^{3}+b\left(x_{0}^{\prime *}\right)^{2}\left|y_{0}^{\prime *}\right|,
$$

where $a$ and $b$ are always positive, the expected value

$$
\langle|\overline{\Delta y}|\rangle=2 \sqrt{\frac{2}{\pi} a}\left(\frac{\epsilon_{y}}{\beta_{y}^{*}}\right)^{3 / 2}+\sqrt{\frac{2}{\pi} b}\left(\frac{\epsilon_{x}}{\beta_{x}^{*}}\right)\left(\frac{\epsilon_{y}}{\beta_{y}^{*}}\right)^{1 / 2}
$$

as defined in Eq. (17) is calculated as 2 times the integral from zero to infinity because of the absolute value. This has the advantage of measuring better the change in the beam core, as the beam tails have a lower effect with respect to $\sigma_{2 \mathrm{D} \text {-Oide }}$ and could better represent the impact in the luminosity. For this reason, it is further referred to as

$$
\begin{aligned}
\sigma_{\Delta \mathrm{core}}^{2} & =\langle|\overline{\Delta y}|\rangle^{2} \\
& =\frac{8}{\pi} a^{2}\left(\frac{\epsilon_{y}}{\beta_{y}^{*}}\right)^{3}+\frac{2}{\pi} b^{2}\left(\frac{\epsilon_{x}}{\beta_{x}^{*}}\right)^{2} \frac{\epsilon_{y}}{\beta_{y}^{*}}+\frac{8}{\pi} a b\left(\frac{\epsilon_{y}}{\beta_{y}^{*}}\right)^{2} \frac{\epsilon_{x}}{\beta_{x}^{*}} .
\end{aligned}
$$

Figure 4 shows the comparison between the three estimations of the beam size contribution $\left(\sigma_{\text {Oide }}, \sigma_{2 \mathrm{D}-\text { Oide }}\right.$, and $\left.\sigma_{\Delta \text { core }}\right)$ as a function of the vertical beta function at the IP. The vertical line superimposed on Fig. 4 corresponds to

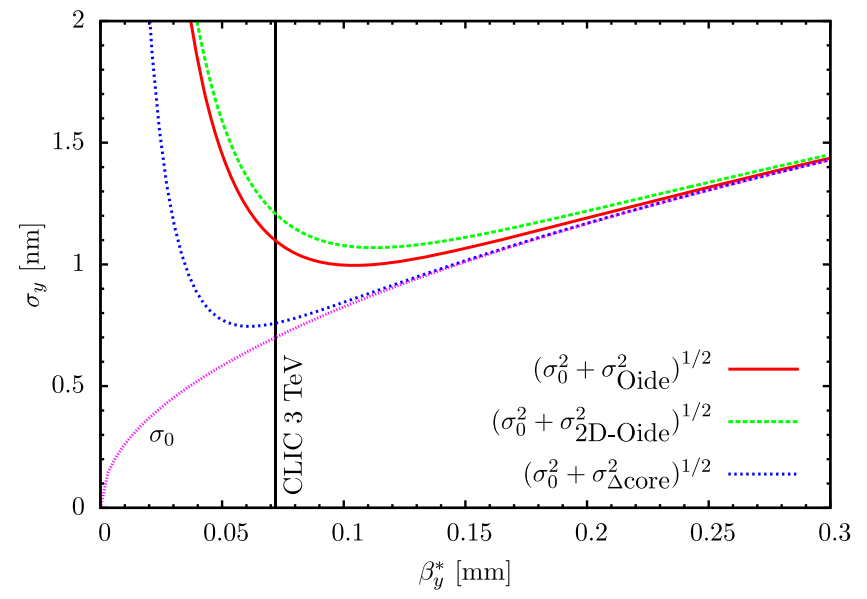

FIG. 4. Beam size as a function of $\beta_{y}^{*}$. The current design value for CLIC $3 \mathrm{TeV}$ is $\beta_{y}^{*}=0.07 \mathrm{~mm}$. $\beta_{y}^{*}=0.07 \mathrm{~mm}$, the current design in CLIC $3 \mathrm{TeV}$. It shows how the minimum beam size achievable is limited by the synchrotron radiation in the final quad and, particularly, how close the CLIC $3 \mathrm{TeV}$ design is to the minimum imposed by $\sigma_{\Delta \text { core }}$.

A detailed approximation of the impact of radiation on luminosity is done in [11] by calculating an effective beam size. This effective beam size was compared to $\sigma_{\Delta \text { core }}$ with their parameters, and both agree well down to the minimum achievable beam size and respective $\beta_{y}^{*}$. For $\beta_{y}^{*}$ below the minimum beam size, $\sigma_{\Delta \text { core }}$ gives an intermediate value between $\sigma_{\text {Oide }}$ (referred as Gaussian in [11]) and the effective beam size; however, the validity of the models with such low $\beta_{y}^{*}$ might not hold.

\section{B. Simulation}

Particle tracking from the entry of QD0 to the IP for CLIC $3 \mathrm{TeV}$ with and without radiation, using PLACET [12], allows one to compute the effects of radiation on the sixdimensional phase space. Figure 5 shows the particle transverse distribution at the IP with and without radiation.

Figure 6 shows tracking results of $\Delta y=y_{\text {rad }}-y_{\text {norad }}$ versus the particle angle $y_{0}^{\prime}$ at the IP for two different horizontal emittances. It has been included to illustrate the radiation effect for the nominal normalized horizontal beam emittance, $\epsilon_{N x}=660 \mathrm{~nm}$, and the case when the horizontal beam size $\sigma_{x}$ is negligible when compared to the vertical beam size which is achieved by reducing the horizontal emittance, $\epsilon_{N x}=10^{-4} \epsilon_{N y}$, i.e., $\epsilon_{N x}=2 \mathrm{pm}$. The horizontal beta Twiss parameter at the IP is the same in both trackings: $\beta_{x}^{*}=6.9 \mathrm{~mm}$.

Fitting results of $\Delta y$ for the two previously mentioned cases are compared in Table II with the analytical evaluation of Eqs. (13)-(16).

In the case of $\epsilon_{N x}=2 \mathrm{pm}$, i.e., a negligible effect of the defocusing plane, the result of $a$ differs by $6 \%$ between the theory and tracking results, while the result of $b \epsilon_{N x} /\left(\gamma \beta_{x}\right)$ shows an unexpected component from tracking. In the case of $\epsilon_{N x}=660 \mathrm{~nm}$, i.e., when the defocusing plane is not negligible, the result of $a$ differs by $-6 \%$ between the theory

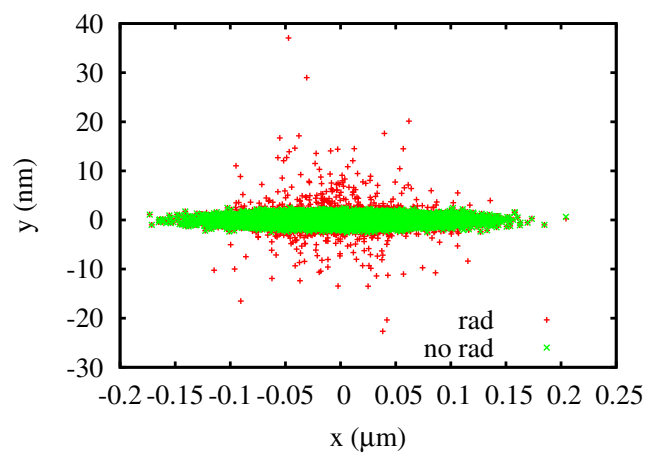

FIG. 5. CLIC $3 \mathrm{TeV}$ beam at the IP after tracking through QD0 with and without radiation. 


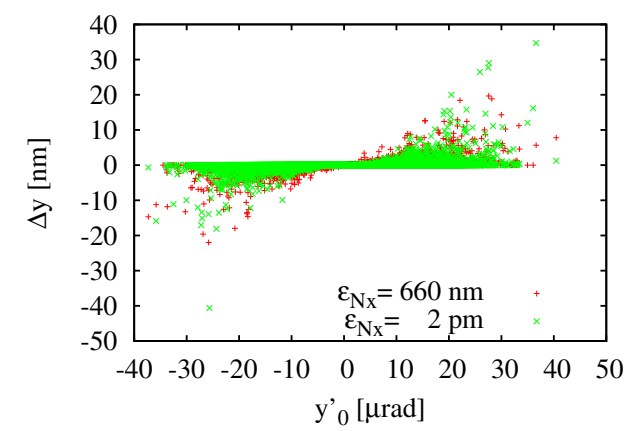

FIG. 6. Correlation between the phase space coordinates $\Delta y, y^{\prime}$ for CLIC $3 \mathrm{TeV}$ from particle tracking with two different horizontal emittances.

and tracking results, while the result of $b \epsilon_{N x} /\left(\gamma \beta_{x}\right)$ shows $-14 \%$ difference. In addition, there is an unexpected $11 \%$ variation in the fitting results of the cubic component $a$ caused by the change of the horizontal emittance in tracking.

The contribution to the beam size $\left\langle\overline{(\Delta y)^{2}}\right\rangle$ from the two theoretical expressions ( $\sigma_{\text {Oide }}$ and $\left.\sigma_{2 \mathrm{D}-\text { Oide }}\right)$ and the particle tracking are compared in Table III, where the errors included in the $\sigma_{2 \mathrm{D} \text {-Oide }}$ results come from numerical precision in the integration of Eq. (19).

The case of $\epsilon_{N x}=2 \mathrm{pm}$ shows that $\sigma_{\text {Oide }}$ and $\sigma_{2 \mathrm{D} \text {-Oide }}$ agree within the numerical precision achieved. The result from tracking is $6 \%$ above the theoretical values. On the other hand, the contribution to radiation calculated from $\sigma_{2 \mathrm{D} \text {-Oide }}$ with the nominal horizontal emittance $\epsilon_{N x}=$ $660 \mathrm{~nm}$ agrees well with the tracking result.

At the moment, the differences between the theory and tracking results for these cases have been attributed to limitations in the particle tracking and radiation simulations.

Comparing $\sigma_{2 \mathrm{D}-\text {-Oide }}$ to $\sigma_{\text {Oide }}$ from Table III, it is possible to conclude that including the second dimension has an important impact of $11 \%$ in the final IP vertical beam size in CLIC $3 \mathrm{TeV}$ because of the additional $17 \%$ beam size contribution from radiation.

\section{Mitigating the impact on the beam size by a nonlinear corrector scheme}

An ideal compensation system would remove the position change due to radiation $\Delta y=y_{\text {rad }}-y_{\text {norad. In this }}$ case, it is possible to remove the average effect due to

TABLE II. Coefficients from fitting tracking results and theory Eq. (13): $y_{0}^{\prime}$ in $\left[10^{-5} \mathrm{rad}\right]$ units.

\begin{tabular}{llll}
\hline \hline & & \multicolumn{2}{c}{$a$} \\
$\overline{\Delta y}$ & & {$\left[10^{-11} \mathrm{~m}\right]$} & $\begin{array}{c}b \epsilon_{N x} /\left(\gamma \beta_{x}\right) \\
{\left[10^{-11} \mathrm{~m}\right]}\end{array}$ \\
\hline$\epsilon_{N x}=2 \mathrm{pm}$ & Theory & 9.0 & 0 \\
& Tracking & $9.5 \pm 0.1$ & $-1.3 \pm 0.3$ \\
$\epsilon_{N x}=660 \mathrm{~nm}$ & Theory & 9.0 & 6.3 \\
& Tracking & $8.5 \pm 0.1$ & $5.4 \pm 0.3$ \\
\hline \hline
\end{tabular}

TABLE III. Contribution to beam size from radiation evaluated by $\sigma_{\text {Oide }}, \sigma_{2 \mathrm{D}-\text {-Oide }}$, and tracking.

\begin{tabular}{lcl}
\hline \hline & $\left\langle\overline{(\Delta y)^{2}}\right\rangle^{1 / 2}$ & {$[\mathrm{~nm}]$} \\
\hline$\epsilon_{N x}=2 \mathrm{pm}$ & $\sigma_{\text {Oide }}$ & 0.87 \\
& $\sigma_{2 \text { D-Oide }}$ & $0.87 \pm 0.03$ \\
& Tracking & 0.92 \\
$\epsilon_{N x}=660 \mathrm{~nm}$ & $\sigma_{2 \text { D-Oide }}$ & $1.02 \pm 0.03$ \\
& Tracking & 1.00 \\
\hline \hline
\end{tabular}

energy loss $\overline{\Delta y}$, because it correlates with the particle angle in the vertical plane $y_{0}^{\prime *}$.

For CLIC $3 \mathrm{TeV},\left\langle(\overline{\Delta y})^{2}\right\rangle^{1 / 2}=0.40 \mathrm{~nm}$, composed of $0.11 \mathrm{~nm}$ from the linear and $0.34 \mathrm{~nm}$ from the cubic components in $y_{0}^{\prime *}$. Using the expression

$$
\frac{\sqrt{\sigma_{2 \text { D-Oide }}^{2}-\left\langle(\overline{\Delta y})^{2}\right\rangle}}{\sigma_{2 \text { D-Oide }}},
$$

there is a possible ideal $8 \%$ reduction in the contribution to the Oide effect by removing the correlation. If only one of the two components is removed, then removing the linear component corresponds to $1 \%$ reduction, while removing the cubic components is $6 \%$ reduction in the contribution to beam size due to radiation.

Several possibilities arise to achieve the mitigation of the beam size growth; in particular, the beam size components due to fringe fields [13] show similarities with the components in $\overline{\Delta y}$, pointing to the possibility to tune the lattice elements to compensate the effect of radiation, possibly using various octupoles.

In this article, one possible correction scheme is tested consisting in the addition of one octupole magnet $\mathrm{C} 0$ in front of the strong focusing quadrupole, placed as in Fig. 7.

The kick given by the corrector, $\Delta y_{c 0}^{\prime}$, changes the vertical position at the IP as $\Delta y_{c 0}=L_{c 0} \Delta y_{c 0}^{\prime}$, where $L_{c 0}$ is the distance from the IP to $\mathrm{C} 0$. The displacement induced by the corrector must have opposite direction to the effect of radiation, $\Delta y_{c 0}=-\overline{\Delta y}$; therefore,

$$
\Delta y_{c 0}^{\prime}=-\frac{\overline{\Delta y}}{L_{c 0}} .
$$

The cubic and linear components of $\overline{\Delta y}$ suggest the use of an octupolar magnet as a corrector; therefore, the kick given by an upright octupolar magnet is given by

$$
\begin{aligned}
& x^{\prime}=-\frac{1}{6} k_{3}\left(x^{3}-3 x y^{2}\right), \\
& y^{\prime}=\frac{1}{6} k_{3}\left(3 x^{2} y-y^{3}\right),
\end{aligned}
$$

where $k_{3}$ is the octupole strength. The difference in sign between the linear and cubic components when comparing Eq. (26) with Eq. (13) means that only one out of the two 


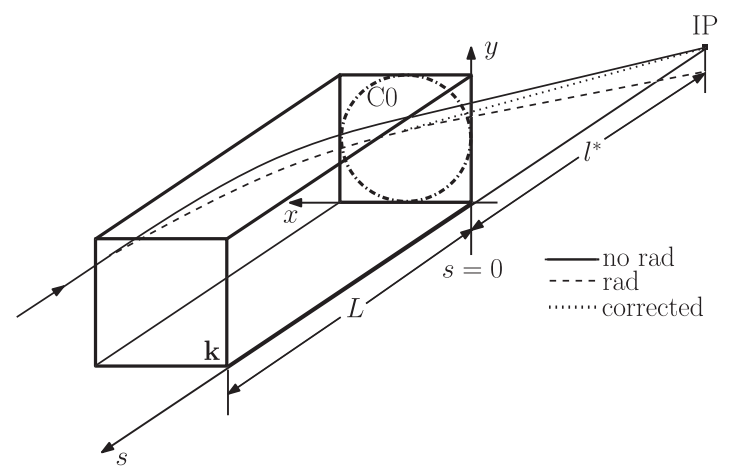

FIG. 7. The solid and dashed lines represent the particle trajectory without and with radiation, respectively. The dotted line represents the correction of the particle trajectory given by $\mathrm{C} 0$.

could be removed from the beam size by an octupolar corrector, limiting the possible mitigation in this simple approach.

The optimum location of the octupolar corrector is in front of the focusing magnet, where the vertical beam size is large and the horizontal beam size is small, which is achieved when maximizing the $\beta_{y} / \beta_{x}$ ratio. Figure 8 shows the horizontal and vertical $\beta$ functions for the CLIC $3 \mathrm{TeV}$ in the FD region and their ratio, where $\beta_{x}=5.3 \times 10^{3} \mathrm{~m}$ and $\beta_{y}=3.3 \times 10^{5} \mathrm{~m}$ at $\mathrm{C} 0$.

Knowing the location of the corrector and matching the kicks in Eqs. (24) and (26), it is possible to obtain the two values for the octupole strength $k_{3}$, one to cancel the linear and another to cancel the cubic component:

$$
\begin{aligned}
& \text { linear: } k_{3}=-\frac{2 b}{L_{c 0}^{4}}, \\
& \text { cubic: } k_{3}=\frac{6 a}{L_{c 0}^{4}},
\end{aligned}
$$

where $x_{0}^{\prime *}=x / L_{c 0}$ and $y_{0}^{\prime *}=y / L_{c 0}$ have been used. For CLIC $3 \mathrm{TeV}$ and $L_{c 0}=3.5 \mathrm{~m}$, the results of Eqs. (27) and (28) are $k_{3}=-2600 \mathrm{~m}^{-4}$ and $k_{3}=3600 \mathrm{~m}^{-4}$, respectively.

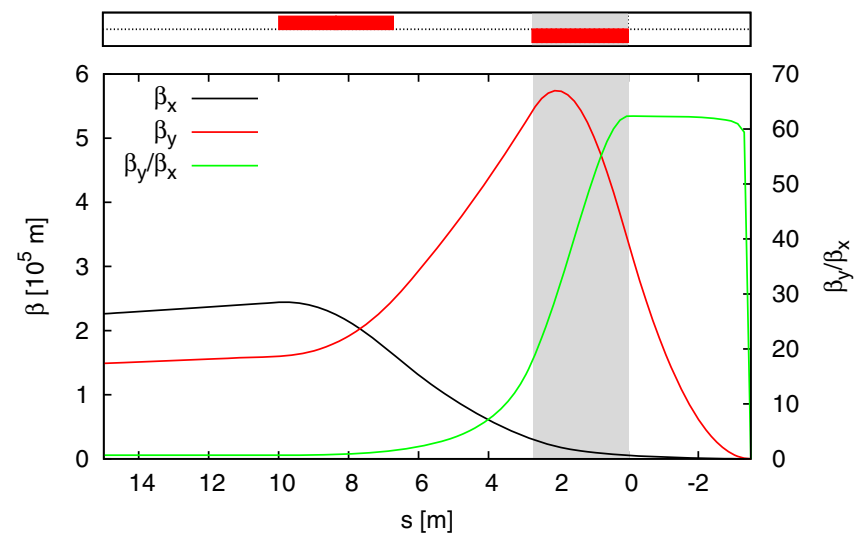

FIG. 8. $\beta$ functions and $\beta_{y} / \beta_{x}$ ratio for CLIC $3 \mathrm{TeV}$ FD, QF1, and QD0 in red on top. The dark area is occupied by QD0, and the IP is at $s=-3.5 \mathrm{~m}$.
TABLE IV. Effect of the octupolar corrector on the beam size,

\begin{tabular}{|c|c|c|c|c|c|}
\hline & \multirow{2}{*}{$\begin{array}{c}k_{3} \\
{\left[\mathrm{~m}^{-4}\right]}\end{array}$} & \multirow{2}{*}{$\begin{array}{c}\sigma_{x} \\
{[\mathrm{~nm}]}\end{array}$} & \multirow{2}{*}{$\begin{array}{c}\sigma_{y} \\
{[\mathrm{~nm}]}\end{array}$} & $L_{\text {tot }}$ & $L_{\text {peak }}$ \\
\hline & & & & \multicolumn{2}{|c|}{$\left[10^{34} \mathrm{~cm}^{-2} \mathrm{~s}^{-1}\right]$} \\
\hline NO RAD & 0 & 47.45 & 0.69 & 7.7 & 2.9 \\
\hline RAD & 0 & 47.45 & 1.18 & 7.5 & 2.7 \\
\hline RAD & 3900 & 47.45 & 1.13 & 7.4 & 2.7 \\
\hline
\end{tabular}
total luminosity, and peak luminosity.

In order to try this octupole mitigation in simulations, a CLIC $3 \mathrm{TeV}$ nominal beam with no energy spread is generated at the IP and tracked back to the entrance of QD0 without radiation with the corrector off. This beam is used to study the Oide effect mitigation in QD0 using the previously mentioned scheme by tracking to the IP with radiation. The procedure consists in setting the best octupole strength for a $10 \mathrm{~mm}$ length $\mathrm{C} 0$.

Results from the tracking to the IP with and without radiation with the corrector off, in Table IV, show that the Oide effect contribution to the vertical beam size affects very little the total and peak luminosities, $L_{\text {tot }}$ and $L_{\text {peak }}$, obtained with Guinea PIG ++ [14].

The best result obtained with the octupole corrector is a vertical beam size reduction by $(-4.3 \pm 0.5) \%$, equivalent to a $6 \%$ reduction in the Oide effect contribution to beam size, which agrees with the removal of the cubic component on the beam size.

The $\mathrm{C} 0$ strength from tracking is positive, also indicating the correction of the cubic component. However, it is $8 \%$ bigger than the theoretical value. This has been attributed to limitations in the simulation and the strength optimization.

\section{CONCLUSIONS}

Synchrotron radiation in the final quadrupole sets a lower limit in the beam size, which is particularly relevant for CLIC $3 \mathrm{TeV}$. This is normally mitigated by increasing the length of the magnet; nevertheless, this produces larger chromatic and geometric aberrations. Previous results considered only the effect of the second moment of the radiation in the focal plane of the quadrupole.

A new value, $\sigma_{2 \mathrm{D}-\text { Oide }}$, has been derived to include the radiation effect of both the focusing and defocusing planes of the quad showing an increase of $17 \%$ in the contribution to beam size. The theory shows good agreement with simulations using the tracking code PLACET.

The variation in the beam core, $\sigma_{\Delta \text { core }}$, has been defined as a workaround to the limitations in the analytic calculation of 2D-Oide and could provide better estimation of the impact in the luminosity. It has been shown that the current CLIC $3 \mathrm{TeV} \beta_{y}^{*}$ lies close to the minimum imposed by this parameter.

A closer insight into the beam correlation induced by synchrotron radiation at the IP has revealed a way to mitigate 
the 2D-Oide effect with octupolar magnets. In general, the 2D-Oide average geometric aberrations can be included in codes like MAPCLASS [6] to mitigate all aberrations simultaneously. The simple case of an octupole is calculated and simulated giving a vertical beam size reduction of $(4.3 \pm 0.5) \%$, with little or negative impact on luminosity.

\section{ACKNOWLEDGMENTS}

This work has been financially supported by The French National Center for Scientific Research (CNRS) and The European Organization for Nuclear Research (CERN).

[1] K. Oide, Synchrotron-Radiation Limit on the Focusing of Electron Beams, Phys. Rev. Lett. 61, 1713 (1988).

[2] O. Blanco, R. Tomas, and P. Bambade, Technical Reports No. CERN-OPEN-2014-051 and No. CLIC-Note-1049, CERN, Geneva, 2014.

[3] R. Tomás, Nonlinear optimization of beam lines, Phys. Rev. Accel. Beams 9, 081001 (2006).

[4] R. Tomás, Technical Reports No. AB-Note-2006-017 and No. CERN-AB-Note-2006-017, CERN, Geneva, 2007.

[5] D. Martinez, A. Rosam, R. Tomas, and R. De Maria, Technical Reports No. CERN-ATS-Note-2012-087 TECH, CERN, Geneva, 2012.

[6] MAPCLASs2, https://github.com/pylhc/MapClass2.
[7] C. Adolphsen et al., arXiv:1306.6353, Technical Reports No. ANL-HEP-TR-13-20, No. BNL-100603-2013-IR, No. IRFU-13-59, No. CERN-ATS-2013-037, No. Cockcroft-13-10, No. CLNS-13-2085, No. DESY-13-062, No. FERMILAB-TM-2554, No. IHEP-AC-ILC-2013-001, No. ILC-REPORT-2013-040, No. INFN-13-04-LNF, No. JAI-2013-001, No. JINR-E9-2013-35, No. JLAB-R2013-01, No. KEK-Report-2013-1, No. KNU-CHEP-ILC2013-1, No. LLNL-TR-635539, No. SLAC-R-1004, and No. ILC-HiGrade-Report-2013-003, KEK, Geneva, 2013. See also http://www.linearcollider.org/ILC/TDR. The full list of signatories is inside the report.

[8] M. Aicheler, P. Burrows, M. Draper, T. Garvey, P. Lebrun, K. Peach, N. Phinney, H. Schmickler, D. Schulte, and N. Toge, Technical Reports No. CERN-2012-007, No. SLACR-985, No. KEK-Report-2012-1, No. PSI-12-01, and No. JAI-2012-001, CERN, Geneva, 2012.

[9] M. Sands, Report No. SLAC-R-121, SLAC, 1970.

[10] SLAC, Zeroth order design report for the Next Linear Collider, Report No. SLAC-R-474, Vols. 1 and 2, 1996.

[11] K. Hirata, K. Oide, and B. Zotter, Synchrotron radiation limit of the luminosity in Tev linear colliders, Phys. Lett. B 224, 437 (1989).

[12] PLACET, https://clicsw.web.cern.ch/clicsw/.

[13] M. Patecki and R. Tomás, Effects of quadrupole fringe fields in final focus systems for linear colliders, Phys. Rev. Accel. Beams 17, 101002 (2014).

[14] D. Schulte, Reports No. CERN-PS-99-014-LP and No. CLIC-Note-387, CERN, Geneva, 1999. 